\title{
Intravenous Injection of Autologous Bone Marrow Derived Mononuclear Cells in Ischemic Stroke Patients in Iraq
}

\author{
Abdul Majeed Alwan Hammadi ${ }^{1}$ and Fizel Abbas Al-himyari ${ }^{2}$ \\ ${ }^{1}$ Department of Medicine, Al-Yermouk Teaching Hospital, Iraq \\ ${ }^{2}$ Department of Neurology, Babylon Medical College, Iraq \\ Received: May 17, 2017; Published: May 31, 2017 \\ *Corresponding author: Abdul Majeed Alwan Hammadi, Medicine Department, Al-Yermouk Teaching Hospital, Baghdad, Iraq \\ Fizel Abbas Al-himyari, Neurology Department, Babylon Medical College, Hillah, Iraq
}

\begin{abstract}
Stroke is a leading cause of adult disability worldwide and the second highest cause of death in the world. Mortality rate is about $20 \%$ and another third are left with permanent disability. Treatments for stroke are based on methods that restore the flow of blood to the brain, such as clot-busting enzymes, surgery, and drugs for thinning blood, stopping clots and protecting neurons. Scientists believe that mesenchymal stem cell protection may be due to secretion of growth factors that promote growth of new neurons, regulate the immune system and stimulate the formation of new blood vessel, Or due to neurogenesis. Autologous bone marrow derived mononuclear cells with closed method is very near to minimal manipulation and low risk procedure.

In this study, we aspirated $100 \mathrm{cc}$ (mean volume) of bone marrow from 50 Iraqi adult patients (age ranges 34-86 years) (12 females 38 male) after filtration we injected $40 \mathrm{cc}$ volume intravenously, mononuclear cell count was $=5-6 \times 107$ per product. The time from diagnosis till procedure performance was (3months- 5 years), The effect of intravenous administration of autologous bone marrow mononuclear cells (BMMNCs) lead to improvement in European scale for stroke (+4-20) in 28 patients out of $50(56 \%)$ in 4-8 weeks ). This study shows the positive effect of autologous bone marrow derived mononuclear cells in the functional recovery of adult patients with chronic ischemic stroke.
\end{abstract}

Keywords: Autologous; Bone Marrow; Mononuclear Cells; Stroke; Intravenous

\section{Introduction}

Stroke is a leading cause of adult disability worldwide and the second highest cause of death in the world. Mortality rate is about $20 \%$ and another third are left with permanent disability. The majority of strokes (85\%) are ischemic, while $15 \%$ is hemorrhagic due to a rupture of a brain artery causes bleeding into the brain tissue. Risk factors for stroke include high blood pressure, irregular heartbeat, diabetes, smoking, carotid artery narrowing, high blood cholesterol, increasing age, and lifestyle factors (exercise, diet and alcohol).

Preventive measures include a diet low in fat and sodium, controlling blood pressure and alcohol intake, maintaining healthy weight, and regular exercise. Treatments for stroke are based on methods that restore the flow of blood to the brain, such as clotbusting enzymes, surgery, and drugs for thinning blood, stopping clots and protecting neurons [1,2]. Local Stem cells might be activated or transplanted from the same patient or another healthy one or from embryonic or cord blood sources [3]. There are many sources of stem cells used clinically to treat ischemic stroke including autologous bone marrow derived or adipose derived stem cells or cultured mesenchymal cells, neural cells derived from embryo, or cord blood cells induced pluripotent stem cells, and Wharton jelly mesenchymal cells. The trials underway are providing preliminary proof of the safety and potential effectiveness of the treatment, but there is still a long way to go before they show significant clinical changes for patients [4]. Safety issues, cell tracking and effectiveness are very vital issues in validating stem cell translational therapy [5].

Scientists believe that mesenchymal stem cell protection may be due to secretion of growth factors that promote growth of new neurons, regulate the immune system and stimulate the formation of new blood vessel, Or due to neurogenesis [6]. Autologous bone marrow cells without cultivation and minimal manipulation can be injected within hour from bone marrow aspiration and therefore suited for autologous administration at any stage of stroke. In addition risk of malignant transformation and chances of contamination are reduced by using autologous minimal manipulation procedure [7]. In this study, the effect of intravenous administration of autologous bone marrow mononuclear cells (BMMNCs) is tested on the recovery of patients with chronic stroke. 


\section{Patients and Methods}

\section{patients characteristics and selection}

Patient's selection was based on the World Medical Association Declaration of Helsinki: in accordance with the Iraqi guidelines for stem cell therapy. Intervention was performed after gaining written informed consent from all the patients/relatives in case of affection of higher mental functions [8]. A single armed clinical study, 50 consecutive, eligible and consenting patients, aged 34-86 year.12 females and 38male with ischemic stroke involving anterior circulation after 3 months -5 years of onset of stroke were included.

\section{Product preparation and infusion}

Bone marrow aspiration was done under local anesthesia with or without sedation, depending on the individual case. Around $100 \mathrm{~mL}$ of bone marrow was aspirated from the posterior iliac crest after proper sterilization using bone marrow aspiration needle (size according to the patient) and collected in heparinized syringes. Mononuclear cells (MNCs) were obtained by filtration. The isolated MNCs were checked for viability manually and confirmed on cell count machine. The separated MNCs (5-6 × 107) were administered immediately after filtration. The mononuclear cells products were infused into antecubital vein (average $40 \mathrm{cc}$ ). The total mononuclear cell count ranges from $5 \times 107$ to $6 \times 107$ for the whole product. We used the European stroke scale to assess clinical response to treatment [9]. Outcomes measured for safety included immediate reactions after cell infusion and evidence of tumor formation at 6 months to one year. Patients were followed monthly after the procedure for 1 year to determine clinical progress using European stroke scale.

\section{Follow up}

Patients were monitored regularly for any immediate adverse effects in the hospital for 3days after the cell therapy. The patients were advised for regular follow-up at 1 month. During each followup, the patients underwent complete neurological assessment and were monitored for any long-term adverse effects. The patients were followed up for minimum of 6 months.

\section{Results}

50 Iraqi patients diagnosed with chronic stroke (3 months5years) were included in this study. 38 male patients and 12 female with age range of 34-86years, all the patients underwent autologous bone marrow mononuclear cells infusion intravenously. Mononuclear cell counts ranges from 5-6 x10 7 per product with $97 \%$ viability. Three patients had mild fever 24 hours within infusion which responded to simple antipyretics. None of the patients had serious adverse events. Out of 50 patients 28 patients showed improvement in the neurological status with increase in European stroke scale from 4 to 20 (56\%). Time from performing the procedure till getting improvement ranges from 4-16 weeks after autologous bone marrow mononuclear cell infusions. Some patients [2] showed marked improvement in speech in 4 weeks' time they were unable to have one or two words tend to speak with some difficulties. Others [6] had improvement in motor power after neuro rehabilitation that increased the European stroke score markedly.

\section{Discussion}

Stem Cell therapy is proposed to improve functional status in many of the neurological diseases, and there are many studies showing safety of the autologous bone marrow derived mononuclear cells and even autologous bone marrow derived mesenchymal stem cells in cases of brain stroke [10]. It is logically clear that transmitting high number of bone marrow derived mononuclear cells inside the lesion or nearby to it is far better than infusing them to the general circulation by intravenous route, as giving the product intravenously will lead to cell trapping in both lung circulation and in other organs like liver and spleen, but technically speaking and for safety purposes intravenous route is preferable, however in one study it was clear that intravenous route is not less effective than intra-arterial route $[11,12]$.

If improvement occurs in patients with chronic ischemic stroke then this is a clear proof regarding effectiveness of this modality of treatment. In contrast to acute or sub acute ones where natural recovery can be a factor and in this current study we got improvement in $56 \%$ of cases. In regard to safety since our procedure is autologous, in addition it is simple noninvasive and use of these cells does not hold the risk of any graft versus host diseases or tumors. Future studies will solve the best route and type of cells to be used in chronic ischemic stroke.

\section{References}

1. Prasad K, Sharma A, Garg A, Mohanty S, Bhatnagar S, et al. (2014) Intravenous autologous bone marrow mononuclear stem cell therapy for ischemic stroke: a multicentric, randomized trial. Stroke 45(12): 3618-3624.

2. P Dharmasaroja (2009) Bone marrow-derived mesenchymal stem cells for the treatment of ischemic stroke. J Clin Neurosci 16(1): 12-20.

3. Hess DC, Borlongan CV (2008) Stem cells and neurological diseases. Cell Prolif 41(1): 94-114.

4. Hess DC, Borlongan CV (2008) Cell-based therapy in ischemic stroke. Expert Rev Neurother 8(8): 1193-1201.

5. Rosado-de-Castro PH, Schmidt Fda R, Battistella V, Lopes de Souza SA, Gutfilen B, et al. (2013) Biodistribution of bone marrow mononuclear cells after intra-arterial or intravenous transplantation in subacute stroke patients. Regen Med 8(2): 145-155.

6. Pavlichenko N, Sokolova I, Vijde S, Shvedova E, Alexandrov G, et al. (2008) Mesenchymal stem cells transplantation could be beneficial for treatment of experimental ischemic stroke in rats. Brain Res 1233: 203213.

7. Shen LH, Li Y, Chen J, Zacharek A, Gao Q et al. (2006) Therapeutic benefit of bone marrow stromal cells administered 1 month after stroke. Journal of Cerebral Blood Flow and Metabolism 27(1): 6-11.

8. General Assembly of the World Medical Association (2004) World Medical Association Declaration of Helsinki: ethical principles for medical research involving human subjects. J Am Coll Dent 81(3): 14-8.

9. Hantson L, De Weerdt W, De Keyser J, Diener HC, Franke C, et al. (1994) The European Stroke Scale. Stroke 25(11): 2215-2219.

10. Savitz SI, Misra V, Kasam M, Juneja H, Cox CS, et al. (2011) Intravenous autologous bone marrow mononuclear cells for ischemic stroke. Ann Neurol 70(1): 59-69. 
11. Fischer UM, Harting MT, Jimenez F, Monzon-Posadas WO, Xue H, et al. (2009) Pulmonary passage is a major obstacle for intravenous stem cell delivery: the pulmonary first-pass effect. Stem Cells Dev 18(5): 683-692.
12. Yang B, Migliati E, Parsha K, Schaar K, Xi X, et al. (2013) Intra-arterial delivery is not superior to intravenous delivery of autologous bone marrow mononuclear cells in acute ischemic stroke. Stroke 44(12): 3463-3472.

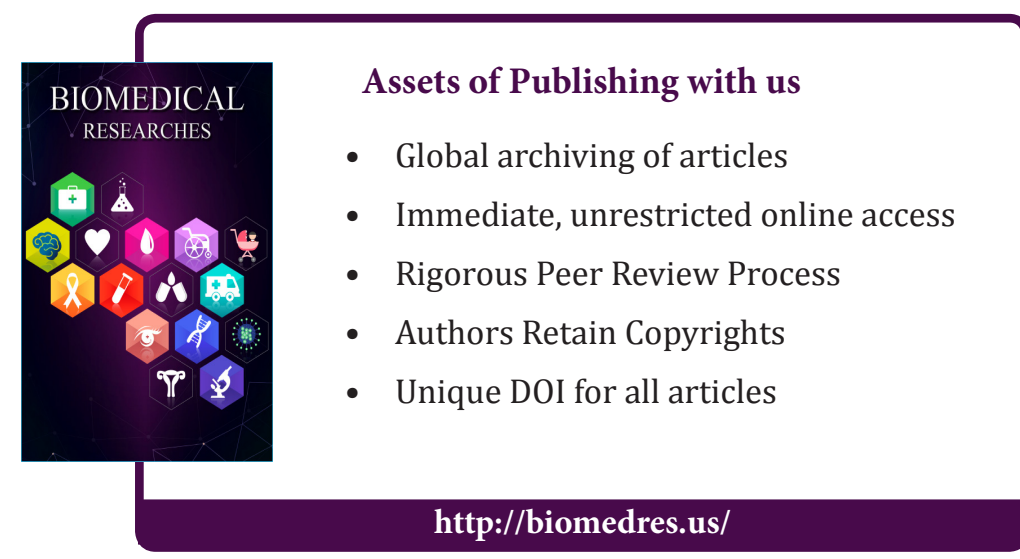

